\title{
Treating adults with acute stress disorder and post-traumatic stress disorder in general practice: a clinical update
}

\author{
David Forbes, Mark C Creamer, Andrea J Phelps, Anne-Laure Couineau, John A Cooper, Richard A Bryant, \\ Alexander C McFarlane, Grant J Devilly, Lynda R Matthews and Beverley Raphael
}

I n Australia, about $65 \%$ of men and $50 \%$ of women are exposed to a potentially traumatic event in their lifetime. ${ }^{1,2}$ Examples of traumatic events include assault and sexual assault, transport accidents, war, and natural disasters. A degree of psychological distress is common in the early aftermath of such traumatic exposure, but when distress persists and interferes with psychosocial functioning, a diagnosis of acute stress disorder (ASD) or post-traumatic stress disorder (PTSD) should be considered. Features of PTSD are shown in Box 1.

The estimated 12-month prevalence of PTSD in the Australian general population is $1.3 \%$, representing around 200000 cases in any one year, and the lifetime prevalence rate (the percentage of people who are diagnosed with the disorder in their lifetime) is likely to be about double this figure. ${ }^{1}$

While a diagnosis of PTSD requires that the symptoms be present for at least a month, ASD is diagnosed between 2 days and 1 month after a traumatic event. There is significant overlap in the diagnostic criteria for these conditions. The diagnoses differ in that ASD requires the experience of several dissociative symptoms not included in PTSD (such as detachment, reduced awareness of surroundings and depersonalisation), while PTSD places greater emphasis on avoidance symptoms by requiring at least three (such as avoidance of thoughts and feelings about the trauma, avoidance of activities, places or people that may act as reminders of the trauma, and passive avoidance symptoms such as emotional numbing and detachment). A growing body of evidence indicates that people with ASD are at high risk of developing PTSD. ${ }^{4}$ However, primarily because of the emphasis on dissociative symptoms, about half of people with PTSD do not meet ASD criteria in the first month after trauma.

In most patients, PTSD is unlikely to present as a stand-alone problem. Comorbid conditions, including depression, other anxiety disorders, and substance misuse, are common. Associated problems include relationship difficulties, excessive anger, and occupational impairment.

\section{Types of symptoms that characterise post-traumatic stress disorder (PTSD)*}

Re-experiencing: Intrusive distressing recollections of the traumatic event; flashbacks; nightmares; intense psychological distress or physical reactions, such as sweating, heart palpitations or panic when faced with reminders of the event (one symptom required).

Avoidance and emotional numbing: Avoidance of activities, places, thoughts, feelings, or conversations related to the event; restricted emotions; loss of interest in normal activities; feeling detached from others; psychogenic amnesia; sense of foreshortened future (three symptoms required).

Hyperarousal: Difficulty sleeping; irritability; difficulty concentrating; hypervigilance; exaggerated startle response (two symptoms required).

* Symptoms must be present for at least a month before a diagnosis can be made. $^{3}$

\section{ABSTRACT}

- General practitioners have an important role to play in helping patients after exposure to severe psychological trauma.

- In the immediate aftermath of trauma, GPs should offer "psychological first aid", which includes monitoring of the patient's mental state, providing general emotional support and information, and encouraging the active use of social support networks, and self-care strategies.

- Drug treatments should be avoided as a preventive intervention after traumatic exposure; they may be used cautiously in cases of extreme distress that persists.

- Adults with acute stress disorder (ASD) and post-traumatic stress disorder (PTSD) should be provided with traumafocused cognitive behaviour therapy (CBT). Eye movement desensitisation and reprocessing (EMDR) in addition to invivo exposure (confronting avoided situations, people or places in a graded and systematic manner) may also be provided for PTSD.

- Drug treatments should not normally replace trauma-focused psychological therapy as a first-line treatment for adults with PTSD.

- If medication is considered for treating PTSD in adults, selective serotonin reuptake inhibitor antidepressants are the first choice. Other new generation antidepressants and older tricyclic antidepressants should be considered as second-line pharmacological options. Monoamine oxidase inhibitors may be considered by mental health specialists for use in people with treatment-resistant symptoms.

MJA 2007; 187: 120-123

The clinical recommendations in this article are based on the recently released evidence-based Australian guidelines for the treatment of adults with acute stress disorder and posttraumatic stress disorder ${ }^{5}$ and the United Kingdom PTSD guidelines. ${ }^{6}$

\section{Screening and assessment in general practice}

Traumatic stress problems present to general practitioners in a variety of ways. Sometimes patients present with expressed concern about their emotional wellbeing after exposure to a traumatic stressor, and may directly report specific symptoms of ASD or PTSD. Such presentations are more common after a recent discrete trauma, such as a motor vehicle accident or an assault. Other patients may present with a greater focus on related problems, such as substance misuse, depression or a specific phobic reaction (such as fear of driving). In such cases, the link to the traumatic experience may not be explicitly made. However, commonly, patients with traumatic stress problems present to GPs with general health complaints, including headaches, gastrointestinal 


\section{Screening questions for post-traumatic stress disorder ${ }^{8}$}

- Do you avoid being reminded of the experience by staying away from certain places, people or activities?

- Have you lost interest in activities that were once important or enjoyable?

- Have you begun to feel more distant or isolated from other people?

- Do you find it hard to feel love or affection for other people?

- Have you begun to feel that there is no point in planning for the future?

- Have you had more trouble than usual falling or staying asleep?

- Do you become jumpy or easily startled by ordinary noise or movements?

Yes $=1$. No $=0$. A score of 4 or higher for these seven questions indicates a possible diagnosis of post-traumatic stress disorder, with a positive predictive value of $71 \%$ and a negative predictive value of $98 \%$, based on a community sample.

problems, rheumatic pains, and skin disorders rather than with expressed concern about the trauma. Indeed, the traumatic event may not be mentioned at all. The distress, stigma and potential shame associated with mental health problems, or with the trauma itself (such as in cases of sexual assault), may prevent some people from mentioning the event.

As a result of such difficulties, traumatic stress conditions are often underdiagnosed in routine clinical settings. ${ }^{7}$ Thus, GPs should consider asking, particularly during their assessment of somatoform or psychosomatic presentations, whether the person has experienced a traumatic event. If post-traumatic mental health problems are suspected, a brief screening measure, such as the one provided in Box 2, should be used to screen for PTSD. Other useful screening instruments are available from the United States Department of Veterans Affairs National Center for Posttraumatic Stress Disorder (http://www.ncptsd.va.gov/ncmain/assessment/ ptsd_screening.jsp).

\section{Management}

The following management advice is based on a recent evidencebased review that analysed the findings of over 69 well controlled psychological and pharmacological treatment studies. This process was consistent with National Health and Medical Research Council (NHMRC) evidence review specifications. ${ }^{9}$ The NHMRC-approved Australian guidelines for the treatment of adults with acute stress disorder and posttraumatic stress disorder ${ }^{5}$ were developed as a result of this process. The guidelines, as well as summary versions for practitioners and consumers, are available at the websites of the NHMRC (http://www.nhmrc.gov.au/publications/) and the Australian Centre for Posttraumatic Mental Health (http:// www.acpmh.unimelb.edu.au).

\section{Management immediately after trauma}

Most people experience some level of distress after a traumatic event, and recover by using their own resources, including their natural coping strategies and social support networks. Thus, formal intervention during the first couple of weeks is not recommended as a routine option, and help should normally be limited to practical and emotional support tailored to individual needs. Recommendations for GPs managing patients in the immediate aftermath of trauma are described in Box 3.

\section{Managing ASD and PTSD}

In patients whose distress is persistent or severe enough to cause significant impairment, a diagnosis of ASD or PTSD should be considered. Thorough clinical assessment should be conducted, including physical, psychological, social, and occupational functioning, as well as assessment of the risk of self-harm, suicide and harm to others. An interview with a family member or friend can be very helpful as part of the assessment process.

PTSD is only one of the potential mental health outcomes after trauma. Thus, assessment should consider other conditions such as depression, other anxiety disorders, and substance misuse, along with the implications for treatment.

A useful self-report measure for assessing PTSD (both initially and to monitor progress throughout treatment) is the PTSD checklist, ${ }^{10}$ described in Box 4.

\section{Treatment for PTSD}

The findings of over 58 well controlled psychological and pharmacological treatment studies are reviewed in the Australian guidelines. ${ }^{5}$ Implications from these findings are summarised below.

- Adults with PTSD should receive trauma-focused psychological interventions from appropriately trained mental health practitioners. These interventions will usually take the form of cognitive behaviour therapy (CBT), or eye movement desensitisation and reprocessing (EMDR) in addition to confronting avoided situations, people or places in a graded and systematic manner (in-vivo exposure).

- Key elements of trauma-focused psychological treatment include:

$>$ confronting the traumatic memory in a controlled and safe environment (imaginal exposure);

> identifying, challenging and modifying biased or distorted thoughts and interpretations about the event and its meaning (cognitive therapy); and

$>$ in-vivo exposure.

\section{Information for general practitioners managing} patients in the immediate aftermath of trauma

- "Psychological first aid" should be undertaken. This involves ongoing monitoring of the patient's mental state, providing general emotional support, ensuring the patient's safety and security, providing practical assistance and information, and encouraging active use of social support mechanisms and selfcare strategies.

- Structured interventions (such as psychological debriefing, with a focus on recounting the traumatic event and ventilation of feelings) should not be offered on a routine basis. However, patients who wish to discuss the experience, and who show a capacity to tolerate the associated distress, should be supported in doing so. The GP should keep in mind the potential adverse effects of excessive ventilation in those who are very distressed or have dissociative symptoms.

- Drug treatments should not be used as a preventive intervention after exposure to trauma (although they may be used cautiously in cases of extreme distress that does not settle, or of severe insomnia). 


\section{Overview of the post-traumatic stress disorder (PTSD) checklist}

- The items on the PTSD checklist (PCL) correspond to the diagnostic criteria of PTSD in the DSM-IV. ${ }^{11}$

- Respondents rate each item from 1 ("not at all") to 5 ("extremely") to indicate the degree to which they have been bothered by that particular symptom over the past month.

- A PCL score of 50 has been identified as a useful cut-off for a likely diagnosis of PTSD.

- The PCL (and its cut-off score) has been validated against goldstandard interview measures, both cross-sectionally and in its capacity to accurately measure change over time. ${ }^{12}$

- Health care providers can obtain a copy of the PCL at <http:// www.ncptsd.va.gov/ncmain/assessment/adult_selfreport.jsp>

- Drug treatments for PTSD should not normally be used in preference to trauma-focused psychological therapy as a routine first-line treatment for adults, either by GPs or by specialist mental health professionals. However, antidepressant medication may be considered for the treatment of PTSD in adults if the patient:

$>$ is unwilling or unable to engage in trauma-focused psychological treatment;

$>$ is not in a sufficiently stable condition to commence traumafocused psychological treatment;

$>$ has not gained significant benefit from a trial of traumafocused psychological treatment; or

$>$ is experiencing severe dissociative symptoms that are likely to be exacerbated by trauma-focused therapy.

- Where medication is considered for treating PTSD in adults, selective serotonin reuptake inhibitor (SSRI) antidepressants should be the first choice for both GPs and mental health specialists. Although sertraline is the most studied of the SSRIs in PTSD, it is hard to argue for the superior efficacy of one SSRI over another.

- Other new generation antidepressants (notably mirtazapine) and the older tricyclic antidepressants should be considered as a second-line pharmacological option. Monoamine oxidase inhibitors may be considered for use by mental health specialists for people with treatment-resistant symptoms.

- When an adult with PTSD has responded to drug treatment, it should be continued for at least 12 months before gradual withdrawal.

- Pharmacological interventions should be considered as an adjunct to psychological treatment where core PTSD or comorbid symptoms are of sufficient severity to impede benefit from psychological treatment alone.

- In the context of comorbidity:

$>$ When PTSD is comorbid with depression, in most cases, the PTSD should be treated first, as depression will often ameliorate as PTSD symptoms reduce. However, when depressive symptoms prevent effective engagement in treatment or are associated with a high risk of suicide, the depression should be managed first.

$>$ When PTSD is comorbid with substance misuse disorders, in patients whose level of substance misuse is low-risk to medium-risk, treat both conditions simultaneously, as the two are likely to interact to maintain each other. Treatment may be less effective if either the PTSD or the substance misuse remains untreated. Substance misuse should be managed before proceeding to the trauma-focused components of PTSD treatment.

\section{Treatment for ASD}

If significant symptoms have been present for more than 2 days but less than a month after trauma, a diagnosis of ASD should be considered. Compared with the large number of treatment studies of PTSD, there have been only a small number of high-quality studies into the psychological and pharmacological treatment of ASD. If ASD is diagnosed, the patient should be offered individual trauma-focused CBT, including exposure and/or cognitive therapy. This treatment should not start until 2 weeks after the traumatic event. Drug treatments should not be used within 4 weeks of symptoms appearing unless the severity of the person's distress cannot be managed by psychological means alone.

\section{When and why to refer}

GPs can do much to assist people recovering from trauma. Reassurance and support, simple information, and advice on selfcare will often be enough to facilitate a natural recovery process. However, if the person's symptoms are severe, and associated with high levels of prolonged distress, or interfere in social and occupational functioning, referral to a mental health care provider (such as a psychiatrist or clinical psychologist) is advisable. Helping the patient to understand what to expect is an important component of referral for specialist care. Trauma-focused psychological treatment (CBT, or EMDR plus in-vivo exposure) can be expected to reduce the symptoms of PTSD, relieve anxiety and depression, and improve quality of life. These therapies can also be effective for people who have experienced prolonged or repeated traumatic events, although more time to establish a trusting therapeutic alliance, more attention to teaching emotional regulation skills, and a gradual approach to exposure therapy may be required in such cases. Interventions that focus purely on reducing symptoms (such as anxiety management), although not as effective when used on their own, are useful adjuncts to trauma-focused treatment. Non-trauma-focused interventions, such as supportive counselling, should not normally be provided to adults with PTSD. Specialist pharmacological treatment for PTSD is best managed by an appropriately experienced psychiatrist, although the continued involvement of patients' GPs in the process is strongly recommended.

Unfortunately, research indicates that only a minority of practitioners apply evidence-based treatments for PTSD in their routine clinical care. ${ }^{13}$ Thus, when referring patients for specialist psychological or pharmacological treatment, it would be prudent to consider whether the specialist practitioner has expertise in evidence-based trauma interventions.

\section{Acknowledgements}

We thank Dr Adele Weston from Health Technology Analysts for support and assistance in the guideline development project in her role as Guideline Assessment Registrar consultant. We also thank the staff of the Australian Centre for Posttraumatic Mental Health for their assistance with this project.

\section{Competing interests}

None identified. 


\section{CLINICAL UPDATE}

\section{Author details}

David Forbes, MClinPsychol, PhD, Associate Professor ${ }^{1}$

Mark C Creamer, PhD, Professor ${ }^{1}$

Andrea J Phelps, MPsych, Senior Lecturer ${ }^{1}$

Anne-Laure Couineau, MA, Senior Lecturer ${ }^{1}$

John A Cooper, MPM, Senior Lecturer ${ }^{1}$

Richard A Bryant, PhD, Professor ${ }^{2}$

Alexander C McFarlane, MD, Professor ${ }^{3}$

Grant J Devilly, PhD, Professorial Fellow ${ }^{4}$

Lynda R Matthews, PhD, Senior Lecturer ${ }^{5}$

Beverley Raphael, MD, Professor ${ }^{6}$

1 Australian Centre for Posttraumatic Mental Health, University of Melbourne, Melbourne, VIC.

2 School of Psychology, University of New South Wales, Sydney, NSW.

3 Centre for Military and Veterans' Health, University of Adelaide,

Adelaide, SA.

4 Brain Sciences Institute, Swinburne University, Melbourne, VIC.

5 Behavioural and Community Health Sciences, University of Sydney, Sydney, NSW.

6 Centre for Disasters and Terrorism, University of Western Sydney, Sydney, NSW.

Correspondence: dforbes@unimelb.edu.au

\section{References}

1 Creamer M, Burgess P, McFarlane AC. Post-traumatic stress disorder: findings from the Australian National Survey of Mental Health and Wellbeing. Psychol Med 2001; 31: 1237-1247.

2 Kessler RC, Sonnega A, Hughes M, Nelson CB. Posttraumatic stress disorder in the national comorbidity survey. Arch Gen Psychiatry 1995; 52: 1048-1060

3 American Psychiatric Association. Practice guideline for the treatment of patients with acute stress disorder and posttraumatic stress disorder.
Arlington, Va: APA, 2004. http://www.psych.org/psych_pract/treatg/pg/ ASD_PTSD_05-15-06.pdf (accessed Jun 2007).

4 Bryant RA. Early predictors of posttraumatic stress disorder. Biol Psychiatry 2003; 53: 789-795.

5 Australian Centre for Posttraumatic Mental Health. Australian guidelines for the treatment of adults with acute stress disorder and posttraumatic stress disorder. Melbourne: ACPMH, 2007. http:// www.acpmh.unimelb.edu.au/Resources/guidelines/ACPMH_FullASDandPTSDGuidelines.pdf (accessed Jun 2007).

6 National Collaborating Centre for Mental Health. Post-traumatic stress disorder. The management of PTSD in adults and children in primary and secondary care. National Clinical Practice Guideline No. 26. London: Gaskell and the British Psychological Society, 2005. http:// www.nice.org.uk/pdf/CG026fullguideline.pdf (accessed Jun 2007).

7 Zimmerman M, Mattia JI. Is posttraumatic stress disorder underdiagnosed in routine clinical settings? J Nerv Ment Dis 1999; 187: 420-428.

8 Breslau N, Peterson EL, Kessler RC, Schultz LR. Short screening scale for DSM-IV posttraumatic stress disorder. Am J Psychiatry 1999; 156: 908-911.

9 National Health and Medical Research Council. How to review the evidence: systematic identification and review of the scientific literature. Canberra: NHMRC, 2000. http://www.nhmrc.gov.au/publications/synopses/cp65syn.htm (accessed Jun 2007)

10 Weathers FW, Litz BT, Herman DS, et al. The PTSD checklist (PCL): reliability, validity, and diagnostic utility. Paper presented at the Annual Meeting of International Society for Traumatic Stress Studies, San Antonio, Tex, October 1993. http://www.pdhealth.mil/library/downloads/ PCL_sychometrics.doc (accessed Jun 2007).

11 American Psychiatric Association. Diagnostic and statistical manual of mental disorders. 4th ed. Washington, DC: APA, 1994.

12 Forbes D, Creamer M, Biddle D. The validity of the PTSD checklist as a measure of symptomatic change in combat-related PTSD. Behav Res Ther 2001; 39: 977-986.

13 Rosen CS, Chow HC, Finney JF, et al. VA practice patterns and practice guidelines for treating posttraumatic stress disorder. J Trauma Stress 2004; 17: 213-222.

(Received 22 Apr 2007, accepted 31 May 2007) 